\title{
Hypoxia-induced autophagy is inhibited by PADI4 knockdown, which promotes apoptosis of fibroblast-like synoviocytes in rheumatoid arthritis
}

\author{
TINGTING FAN $^{1 *}$, CHANGSONG ZHANG $^{2 *}$, MING ZONG $^{1 *}$ and LIEYING FAN ${ }^{1}$ \\ ${ }^{1}$ Department of Clinical Laboratory, Shanghai East Hospital, Tongji University Medical School, Shanghai 200120; \\ ${ }^{2}$ Clinical Oncology Laboratories, Changzhou Tumor Hospital, Medical College of Soochow University, \\ Changzhou, Jiangsu 213032, P.R. China
}

Received November 17, 2016; Accepted September 12, 2017

DOI: $10.3892 / \mathrm{mmr} .2018 .8501$

\begin{abstract}
Impaired apoptosis of rheumatoid arthritis (RA)-fibroblast-like synoviocytes (FLS) is pivotal in the process of RA. Peptidyl arginine deiminase type IV (PADI4) is associated with autoantibody regulation via histone citrullination in RA. The present study aimed to investigate the role of PADI4 in the apoptosis of RA-FLS. FLS were isolated from patients with RA and a rat model. The effects of PADI4 on RA-FLS were investigated in vitro and in vivo. Hypoxia-induced autophagy was induced by $1 \% \mathrm{O}_{2}$ and was detected by immunohistochemical and immunofluorescence analysis; in addition, apoptosis was detected by flow cytometry. RA-FLS obtained from RA rat model exhibited significant proliferation under severe hypoxia conditions. Hypoxia also significantly induced autophagy and elevated the expression of PADI4. Subsequently, short hairpin RNA-mediated PADI4 knockdown was demonstrated to significantly inhibit hypoxia-induced autophagy and promote apoptosis in RA-FLS. The results of these in vitro and in vivo studies suggested that PADI4 may be closely associated with hypoxia-induced autophagy, and the inhibition of hypoxia-induced autophagy
\end{abstract}

Correspondence to: Dr Lieying Fan, Department of Clinical Laboratory, Shanghai East Hospital, Tongji University Medical School, 150 Jimo Road, Shanghai 200120, P.R. China

E-mail: flieying@yeah.net

*Contributed equally

Abbreviations: RA, rheumatoid arthritis; FLS, fibroblast-like synoviocytes; PADI4, peptidyl arginine deiminase type IV; OA, osteoarthritis; ACPA, anti-cyclic citrullinated peptides antibody; IHC, immunohistochemistry; LC3, microtubule-associated protein light chain 3; Atg5, autophagy-related gene 5; HIF-1 $\alpha$, hypoxia-inducible factor- $1 \alpha$

Key words: peptidyl arginine deiminase IV, autophagy, rheumatoid arthritis, apoptosis by PADI4 knockdown may contribute to an increase in the apoptosis of RA-FLS.

\section{Introduction}

Rheumatoid arthritis (RA) is a systemic and chronic inflammatory disease affected by genetic and environmental factors, which leads to joint destruction and dysfunction. Individuals with RA frequently possess autoantibodies against citrullinated peptides, thus indicating that peptidyl arginine deiminase type IV (PADI4) may be associated with RA. It has previously been reported that PADI4 haplotypes associated with susceptibility to RA increase the production of citrullinated peptides as autoantigens, leading to an increased risk of developing RA (1).

PADI4 is localized in the cytoplasm of monocytes, $\mathrm{T}$ and B cells, neutrophils, eosinophils and natural killer cells, and can translocate to the nucleus upon cell activation (2). PADI4 is involved in the post-translational conversion of arginine residues to citrulline. Therefore, it has been suggested that increased translation of variant PADI4 mRNA promotes citrullinated peptide production, which acts as an autoantigen and causes marked adaptive immune responses (3). To date, the association between the PADI4 gene and the severity of RA has been reflected with regards to the correlation between PADI4 haplotypes (or alleles) and serum titer (or positivity) of the anti-cyclic citrullinated peptides antibody (ACPA) (4-6). However, PADI4 may serve an additional role in the occurrence and development of RA, in addition to its role in ACPA formation (7).

A substantial increase in the number of resident synovial cells is an important pathophysiological characteristic in patients with RA (8). One of the key components of invasive synovium is RA fibroblast-like synoviocytes (RA-FLS), which are associated with the development and progression of destructive joint inflammation, and are resistant to receptor-mediated apoptosis at numerous levels, alongside the alteration in mitochondrial pathway-mediated apoptosis, another characteristic of RA $(9,10)$. In addition to apoptosis, autophagy is another important mechanism that regulates cell survival and homeostasis. Numerous mechanisms that contribute to reduced 
apoptosis have been extensively studied, including autophagy, which is modulated by apoptosis $(11,12)$. RA-FLS are resistant to apoptosis, which is induced by apoptotic stimuli; however, the role of apoptosis, as well as autophagy, in the regulation of RA-FLS remains unclear. The present study aimed to provide an overview regarding the participation of PADI4 in the apoptosis of RA-FLS, and its role in the hypoxia-induced autophagy of RA-FLS.

\section{Materials and methods}

Ethical approval. The present study was approved by the Ethics Committee of Tongji University (Shanghai, China) Written informed consent was obtained from all participants prior to the study.

Patients and tissue samples. All the specimens were obtained from patients admitted to Shanghai East Hospital in China from February 2016 to December 2016. Synovial tissues were obtained from 8 patients with RA, including 2 males and 6 females (age range, 48-74 years; median age, 58 years). In addition, synovial tissues were also obtained from 6 patients with osteoarthritis (OA; 3 males and 3 females; age range, 48-77 years; median age, 60 years), who underwent knee arthroscopic or replacement surgery, were recruited to the present study (13). The tissue samples were immediately processed for immunohistochemical analysis. All patients fulfilled the American College of Rheumatology criteria for the diagnosis of RA and OA.

Animal model. A total of 18 inbred female rats (SpragueDawley; age, 7-8 weeks) weighing $\geq 150$ g were purchased from Shanghai SLAC Laboratory Animal Co., Ltd., (Shanghai, China) and were allowed to acclimate for $\geq 1$ week prior to experimentation. The rats were housed in an animal facility and were maintained at $25^{\circ} \mathrm{C}$ (humidity, 60-70\%) under a $12 \mathrm{~h}$ light/dark cycle with ad libitum access to food and water. Bovine type II collagen (CII; Chondrex, Inc., Redmond, WA, USA) was used to induce RA, which was dissolved in $0.05 \mathrm{~mol} / 1$ acetic acid to a final concentration of $2.0 \mathrm{mg} / \mathrm{ml}$, and was vortexed overnight at $4^{\circ} \mathrm{C}$. Subsequently, CII was emulsified with complete Freund's adjuvant (Chondrex, Inc.) at a ratio of $1: 1$. The rats were immunized with $300 \mu \mathrm{l} \mathrm{CII}$ emulsion by subcutaneous injection into the tail root. On day 7 , the rats received a subcutaneous booster injection (300 $\mu \mathrm{l})$ into the tail; the primary injection site was avoided. After 28 days following the induction of the RA model via CII, the rats were sacrificed. All procedures that involved animals were performed in accordance with the institutional animal welfare guidelines of Tongji University (14). The rats were divided into the following groups: i) Control group, in which RA was not induced $(n=6)$ and were treated with saline and an ii) RA group, in which RA was induced via CII $(n=12)$. Rats were examined three times per week.

Cell lines and reagents. Synovial tissues were obtained from the rats; N-FLS were obtained from the control group and RA-FLS were obtained from the RA group. Synovial tissues were minced into pieces of 2 to $3 \mathrm{~mm}$ in size and incubated with 1640 medium (Invitrogen; Thermo Fisher Scientific,
Table I. Sequences of PADI4-short hairpin RNA.

Gene $\quad$ Sequence $\left(5^{\prime} \rightarrow 3^{\prime}\right)$

PADI4

Candidate sequence CCAGAUUUUGGCUAUGUAACUTT Scrambled sequence GACAUUGAGAGAACAUAAUUCTT

PADI4, peptidylarginine deiminase type IV.

Inc., Waltham, MA, USA) containing $10 \%$ FBS in a humidified atmosphere containing $5 \% \mathrm{CO}_{2}$, which was changed every 3-5 days, and non-adherent tissue pieces were carefully removed. FLS from synovial tissues in the rat model were cultured in Dulbecco's modified Eagle's medium (Gibco; Thermo Fisher Scientific, Inc.) supplemented with $10 \%$ fetal bovine serum (FBS; Invitrogen; Thermo Fisher Scientific, Inc.) at $37^{\circ} \mathrm{C}$ in a humidified atmosphere containing $5 \% \mathrm{CO}_{2}$. FLS were grown over 4-6 passages. Subsequently, FLS were cultured in $1 \% \mathrm{O}_{2}$ for $48 \mathrm{~h}$ to induce autophagy and in $20 \% \mathrm{O}_{2}$ for normal conditions.

Adenovirus production and transient transfection. The short hairpin (sh)RNA sequences of PADI4 were designed using Oligoengine 2.0 software (Oligoengine, Seattle, WA, USA) and were verified by nucleotide BLAST searches (https://blast.ncbi.nlm.nih.gov/Blast.cgi?PAGE_ TYPE=BlastSearch). The candidate sequence and the scrambled sequence with no significant homology are listed in Table I. The shRNA sequence or coding sequence (NM_012387.2) of PADI4 was cloned into pHBAd (Shanghai GenecChem Co., Ltd., Shanghai, China) or GV314 adenovirus vectors (Shanghai GeneChem Co., Ltd.) using BamHI/AgeI (Shanghai GeneChem Co., Ltd.), respectively. FLS were seeded $\left(1 \times 10^{4}\right.$ cells/well) in 96 -well plates overnight. A total of $1 \mu \mathrm{g}$ green fluorescent protein (GFP)-microtubule-associated protein light chain 3 (LC3) expressing plasmids, along with $1 \mu \mathrm{g}$ GV314-PADI4 (Ad-PADI4) or $1 \mu \mathrm{g}$ pHBAd-shPADI4 (sh-PADI4) adenovirus vectors, were transiently transfected into rat normal FLS (N-FLS) or RA-FLS, respectively using the Fugene HD transfection reagent (cat. no. 04709705001; Roche Diagnostics, Indianapolis, IN, USA), according to the manufacturer's protocol. Cells were transfected with GFP-LC3, along with Ad-PADI4 or sh-PADI4 adenovirus vectors, for $8 \mathrm{~h}$. At the end of each experiment, autophagy was detected by counting the percentage of GFP-LC3-expressing FLS under a fluorescence microscope (IX71; excitation wavelength was between 460-550 nm; Olympus Corporation, Tokyo, Japan). The cells transfected with GFP-LC3 expressing plasmids along with blank GV314 (Ad-Vector) or pHBAd-scramble shRNA (sh-Vector) adenovirus vectors were used as negative controls. A minimum of 200 cells were counted in each sample. The experiment was conducted in triplicate.

Reverse transcription-quantitative polymerase chain reaction $(R T-q P C R)$. Briefly, total RNA was extracted from synovial tissues and FLS using TRIzol reagent (Invitrogen; Thermo Fisher Scientific, Inc.) according to the manufacturer's protocol. 
First-strand cDNA was synthesized from $2 \mu \mathrm{g}$ total RNA using an AMV reverse transcriptase kit (Fermentas; Thermo Fisher Scientific, Inc.) for $60 \mathrm{~min}$ at $37^{\circ} \mathrm{C}, 5 \mathrm{~min}$ at $85^{\circ} \mathrm{C}$ and $5 \mathrm{~min}$ at $4^{\circ} \mathrm{C}$. The primer sequences are listed in our previous study (13). qPCR was conducted using a SYBR-Green PCR kit (Toyobo Life Science, Osaka, Japan) with the 7300 Real-Time PCR system (Applied Biosystems; Thermo Fisher Scientific, Inc.). The PCR cycling conditions were as follows: $95^{\circ} \mathrm{C}$ for $10 \mathrm{~min}$, followed by 40 cycles at $95^{\circ} \mathrm{C}$ for $15 \mathrm{sec}$ and $60^{\circ} \mathrm{C}$ for $45 \mathrm{sec}$, and a final extension step of $95^{\circ} \mathrm{C}$ for $15 \mathrm{sec}, 60^{\circ} \mathrm{C}$ for $1 \mathrm{~min}, 95^{\circ} \mathrm{C}$ for $15 \mathrm{sec}$ and $60^{\circ} \mathrm{C}$ for $15 \mathrm{sec}$. $\beta$-actin mRNA expression was used an internal control for normalization. The average $\mathrm{C}_{\mathrm{q}}$ values for triplicate reactions were calculated, and relative expression was determined with the comparative $\mathrm{C}_{\mathrm{q}}$ method (15) using average $\mathrm{C}_{\mathrm{q}}$ values.

Cell viability assay. An MTT assay was performed to analyze cell viability. Cells were plated in 96-well plates at $5 \times 10^{3}$ cells $/ 100 \mathrm{ml}$ medium overnight prior to the experiment. The cell viability in each well was examined using MTT colorimetric assay $(5 \mathrm{mg} / \mathrm{ml}$; cat. no. M2003; Sigma-Aldrich; Merck KGaA, Darmstadt, Germany). MTT solution was then added to each well and incubated for $3 \mathrm{~h}$ at $37^{\circ} \mathrm{C} ; 100 \mu \mathrm{l}$ dimethyl sulfoxide was used to dilute the formazan crystals. The optical density value of each sample was measured at $490 \mathrm{~nm}$ using a plate reader. All determinations were carried out in sextuplicate.

Cell apoptosis assay. Cell apoptosis was performed by using Annexin V Apoptosis Detection kit APC (eBioscience; Thermo Fisher Scientific, Inc.). FLS $\left(2 \times 10^{5}\right.$ cells/well) were cultured in 6-well plates until they reached $70-80 \%$ confluence, after which the cells were collected by trypsinization, washed twice with ice-cold Annexin V binding buffer, and stained with $300 \mu \mathrm{l} 1 \mathrm{X}$ binding buffer containing $5 \mu \mathrm{l}$ Annexin $\mathrm{V}$ and $5 \mu \mathrm{l}$ propidium iodide (PI) for $30 \mathrm{~min}$ at room temperature in the dark. Subsequently, $400 \mu 1$ Annexin V binding buffer was added and the cells were analyzed using flow cytometry (BD Biosciences, Franklin Lakes, NJ, USA), according to the manufacturer's protocol; $\geq 30,000$-gated events were acquired from each sample. Early stage apoptotic cells were stained with fluorescein isothiocyanate (FITC) Annexin V, but not PI; whereas late stage apoptotic cells and necrotic cells were stained positively for FITC Annexin V and PI.

$H \& E$, immunohistochemistry (IHC) and immunofluorescence. Briefly, synovial tissues specimens were dehydrated and embedded in paraffin; subsequently, 4- $\mu \mathrm{m}$ tissue sections were cut using a Leica Biosystem Rotary Microtome (Leica Microsystem Nussloch GmbH, Wetzlar, Germany). The sections of the synovial tissues were stained with HE (Richard Allan Scientific Co.; Thermo Fisher Scientific, Inc.). IHC was performed using the standard streptavidin-biotin-peroxidase complex method. The synovial tissue sections were incubated with affinity-purified anti-PADI4 (1:100; cat. no. ab128086; Abcam, Cambridge, UK), anti-autophagy-related gene 5 (ATG5; 1:100; cat. no. ab109490; Abcam), anti-LC3 (1:200; cat. no. 3868) and anti-hypoxia-inducible factor-1 $\alpha$ (HIF-1 $\alpha$ ) antibodies (1:50; cat. no. 79233; Cell Signaling Technology, Inc., Danvers, MA, USA) for $1 \mathrm{~h}$ at room temperature, followed by incubation with a biotin-labeled secondary antibody (1:2,000; cat. no. A0192; Beyotime Institute of Biotechnology, Co., Ltd., Haimen, China) for $1 \mathrm{~h}$ at room temperature. Finally, slides were developed using 3,3-diaminobenzidine (Shanghai Long Island Biotec, Co., Ltd., China) solution, and were counterstained with hematoxylin (BASO, Taipei, Taiwan). The primary antibody was omitted in control experiments. The slides were observed under an identical lightmicroscope (IX70; Olympus Corporation) at a magnification of $\mathrm{x} 200$ or $\mathrm{x} 400$. The specimens were evaluated by two independent observers who were unaware of the clinical information.

For immunofluorescence, FLS were cytospun at $1,000 \mathrm{x} \mathrm{g}$ for $4 \mathrm{~min}$ at $25^{\circ} \mathrm{C}$ and fixed with $4 \%$ paraformaldehyde for $30 \mathrm{~min}$ at $25^{\circ} \mathrm{C}$. Following permeabilization by $0.5 \%$ Triton X-100 (Beijing Solarbio Science \& Technology Co., Ltd., Beijing, China) for $10 \mathrm{~min}$ and blocking by $1 \%$ bovine serum albumin (Beijing Solarbio Science \& Technology Co., Ltd.) for $1 \mathrm{~h}$ at $25^{\circ} \mathrm{C}$. Cells were stained sequentially with the PADI4 (1:100; cat. no. ab128086; Abcam), LC3 (1:200; cat. no. 3868; Cell Signaling Technology, Inc.) or Beclin1 (1:200; cat. no. Ab207612; Abcam) antibody overnight at $4^{\circ} \mathrm{C}$, after blocking with $2 \%$ bovine serum albumin (Sigma-Aldrich; Merck KGaA) in PBS for $1 \mathrm{~h}$. Cells were washed three times with PBS containing $0.1 \%$ Tween-20 and were incubated for $1 \mathrm{~h}$ with a corresponding FITC-conjugated secondary antibody (1:500; cat. no. A0568; Beyotime Institute of Biotechnology, Co., Ltd.) at room temperature. For the identification of cells with nuclear changes typical of apoptosis, cells were stained with $0.1 \mathrm{mg} / \mathrm{ml}$ DAPI for $5 \mathrm{~min}$. The samples were examined under a fluorescence microscope (IX70; Olympus Corporation).

Statistical analysis. All data were generated without knowledge of the clinical status of the samples, and were analyzed using GraphPad Prism software, version 5 (GraphPad Software, Inc., La Jolla, CA, USA). Student's t-test was performed for the analysis of paired and unpaired samples. One-way analysis of variance and a multiple comparisons test (Least Significant Difference test) among three groups were performed. $\mathrm{P}<0.05$ was considered to indicate a statistically significant difference.

\section{Results}

PADI4 promotes the proliferation of FLS in human RA via hypoxia-induced autophagy. In order to investigate the effects of PADI4 on the growth of human FLS in RA, immunostaining was performed in synovial tissues obtained from patients with RA and OA. As presented in Fig. 1A, PADI4 was overexpressed in RA-FLS, and the proliferation of FLS increased in RA-FLS compared with N-FLS. These results indicated that PADI4 may affect the growth of RA-FLS in patients with RA, compared with in patients with OA.

Since apoptosis is often associated with autophagy, the present study aimed to determine whether PADI4 could induce impaired apoptosis of RA-FLS through hypoxia-induced autophagy. HIF-1 $\alpha$ is an important inducible factor responsible for cellular adaptation to low oxygen tension, and is able to induce autophagy; therefore, the present study determined whether HIF-1 $\alpha$ was involved in hypoxia-induced autophagy. As shown in Fig. 1A, HIF-1 $\alpha$ was overexpressed in RA-FLS 


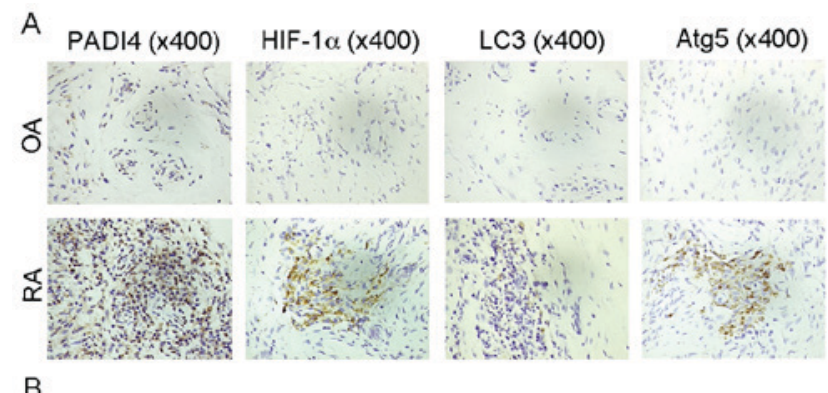

B

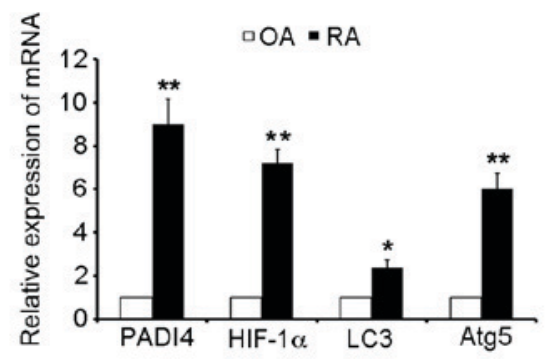

Figure 1. PADI4 and autophagy marker expression in arthritic synovial tissues from patients with RA and OA. (A) Representative images of immunohistochemical staining of PADI4, HIF- $1 \alpha$, LC3 and Atg5 in patients with RA and OA (magnification, $x 400$ ). (B) Relative mRNA expression levels of PADI4, HIF-1 $\alpha$, LC3 and Atg5 in arthritic synovial tissues from patients with RA and OA. Data represent three independent experiments with presented as mean \pm standard deviation. ${ }^{~} \mathrm{P}<0.01 ;{ }^{* *} \mathrm{P}<0.001$. Atg 5 , autophagy-related gene 5; HIF-1 $\alpha$, hypoxia-inducible factor-1 $\alpha$; LC3, microtubule-associated protein light chain 3; OA, osteoarthritis; PADI4, peptidyl arginine deiminase type IV; RA, rheumatoid arthritis.

compared with in OA. In order to analyze the mechanism underlying the autophagic process, IHC was used to monitor and evaluate autophagic activity. LC3 and Atg5 are specific markers of autophagy initiation; therefore, LC3 and Atg5 expression were characterized in resected synovial specimens obtained from patients with RA. The results revealed that LC3 and Atg5 were overexpressed in the RA samples, compared with in the OA tissues (Fig. 1A). Furthermore, it was demonstrated that the mRNA expression levels of PADI4 and markers of autophagy (HIF-1 $\alpha$, LC3 and Atg5) were increased in patients with RA compared with in patients with OA (Fig. 1B). Together, these results suggested that PADI4 may be involved in the response of FLS to hypoxia-induced autophagy.

PADI4 promotes the proliferation of FLS in RA in vivo. Inflammation of the ankles was quantified daily by measuring ankle diameter using the standard caliper method from disease onset to day 28 after the initiation of arthritis (Fig. 2A). At the end of 4 weeks, the animals were sacrificed; the left and right femurs were dissected from the soft tissue, and fixed in $10 \%$ natural buffered formalin for analysis (Fig. 2B). The effects of PADI4 in vivo were evaluated after the initiation of arthritis. PADI4 was overexpressed in synovial tissues in the RA group rats (Fig. $2 \mathrm{C}$ and D). In addition, it was observed that RA-FLS exhibited increased proliferation in the RA group (Fig. 3A and B). These results suggested that PADI4 may have a similar effect on the growth of RA-FLS in rats, similar to in patients with RA.

In vitro PADI4 promotes the proliferation of FLS through hypoxia-induced autophagy. FLS in $1 \% \mathrm{O}_{2}$ were observed
A
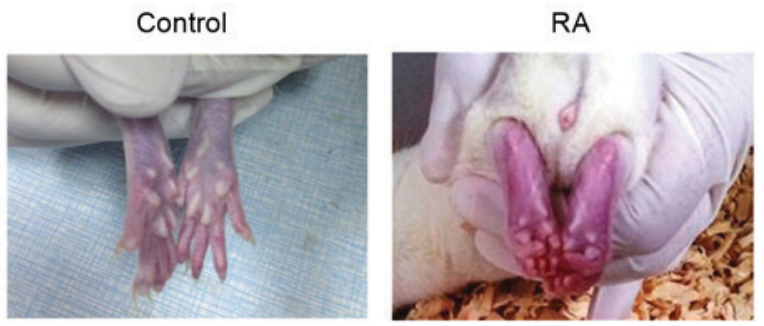

B

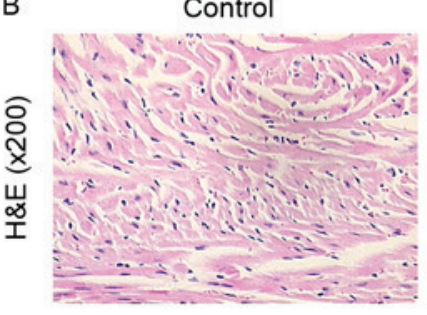

RA

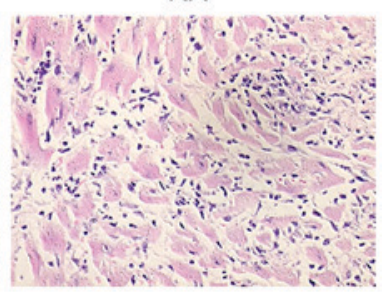

RA

C Control
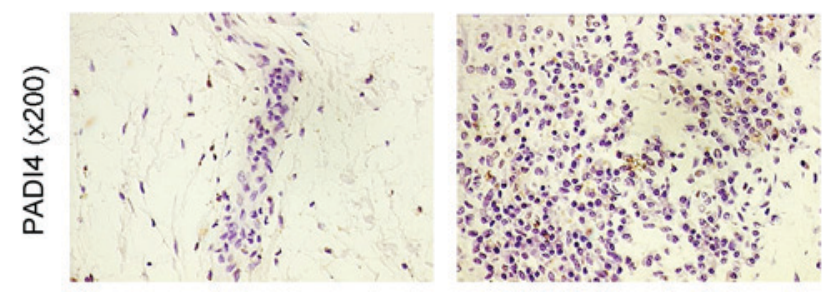

D

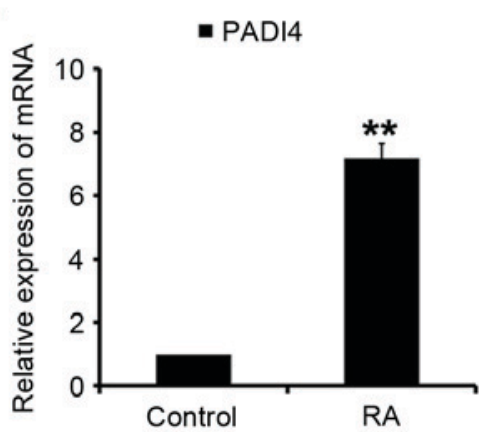

Figure 2. PADI4 expression in arthritic synovial tissues from a rat model of RA. (A) RA was induced via subcutaneous inoculation of RA-FLS into rats. (B) Representative images of H\&E staining in RA and control group samples (magnification, x200). (C) Representative images of PADI4 immunohistochemical staining in RA and control group samples (magnification, x200). (D) Relative mRNA expression levels of PADI4 in arthritic synovial tissues from RA and control group samples. Data represent three independent experiments with presented as mean \pm standard deviation. ${ }^{* *} \mathrm{P}<0.001$. FLS fibroblast-like synoviocytes; H\&E, hematoxylin and eosin; PADI4, peptidyl arginine deiminase type IV; RA, rheumatoid arthritis.

to markedly proliferate in a time-dependent manner (Fig. 3A and B). These results indicated that hypoxia significantly increased RA-FLS proliferation compared with N-FLS proliferation. The effects of 20 and $1 \% \mathrm{O}_{2}$ on PADI4 expression in vitro were evaluated. As shown in Fig. 3C and D, PADI4 was overexpressed in FLS under hypoxia compared with in cells under normoxia. These results suggested that PADI4 may promote the proliferation of RA-FLS through hypoxia.

Hypoxia-induced autophagy in RA-FLS was subsequently analyzed. The results demonstrated that autophagy was significantly increased in the first $24 \mathrm{~h}$ of hypoxia, and was further increased after $48 \mathrm{~h}$ of hypoxia (data not shown). The 
A
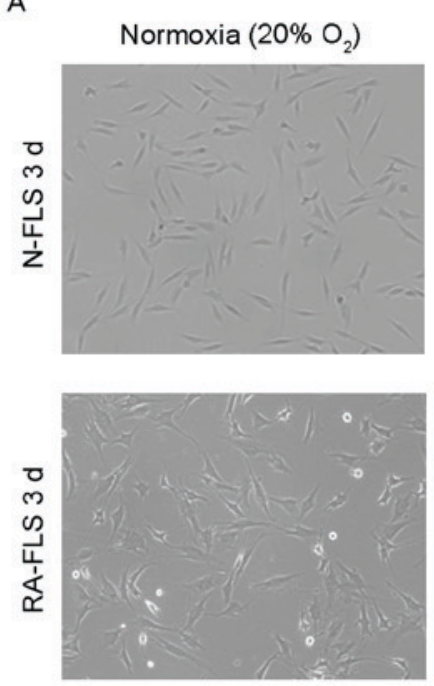

C

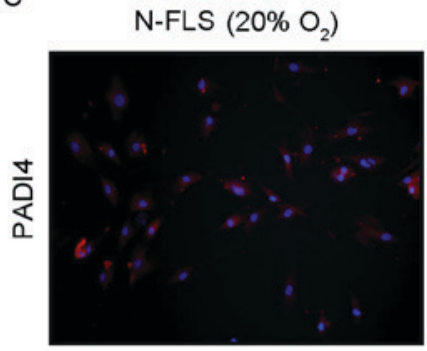

$\mathrm{D}$

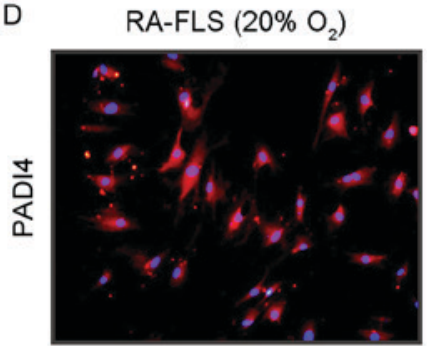

Hypoxia $\left(1 \% \mathrm{O}_{2}\right)$
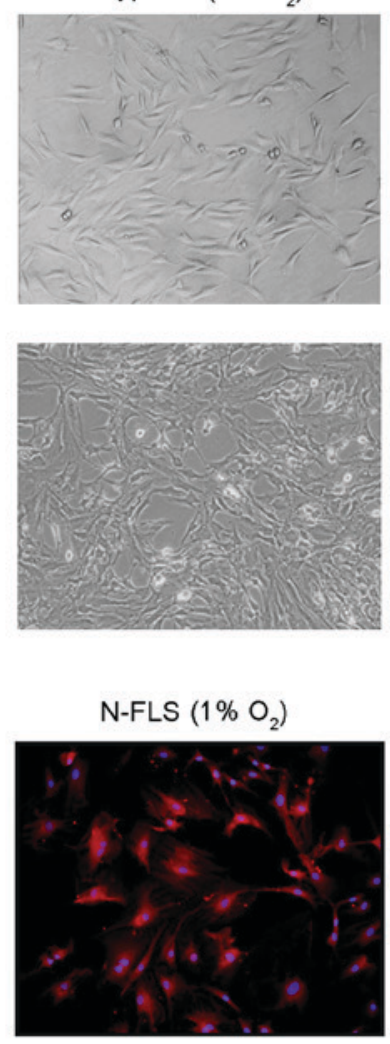

RA-FLS $\left(1 \% \mathrm{O}_{2}\right)$

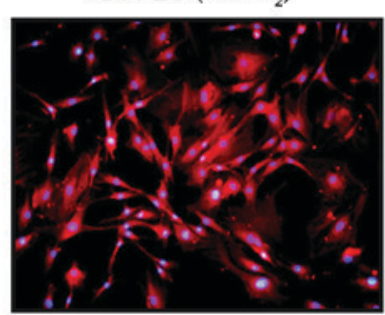

B
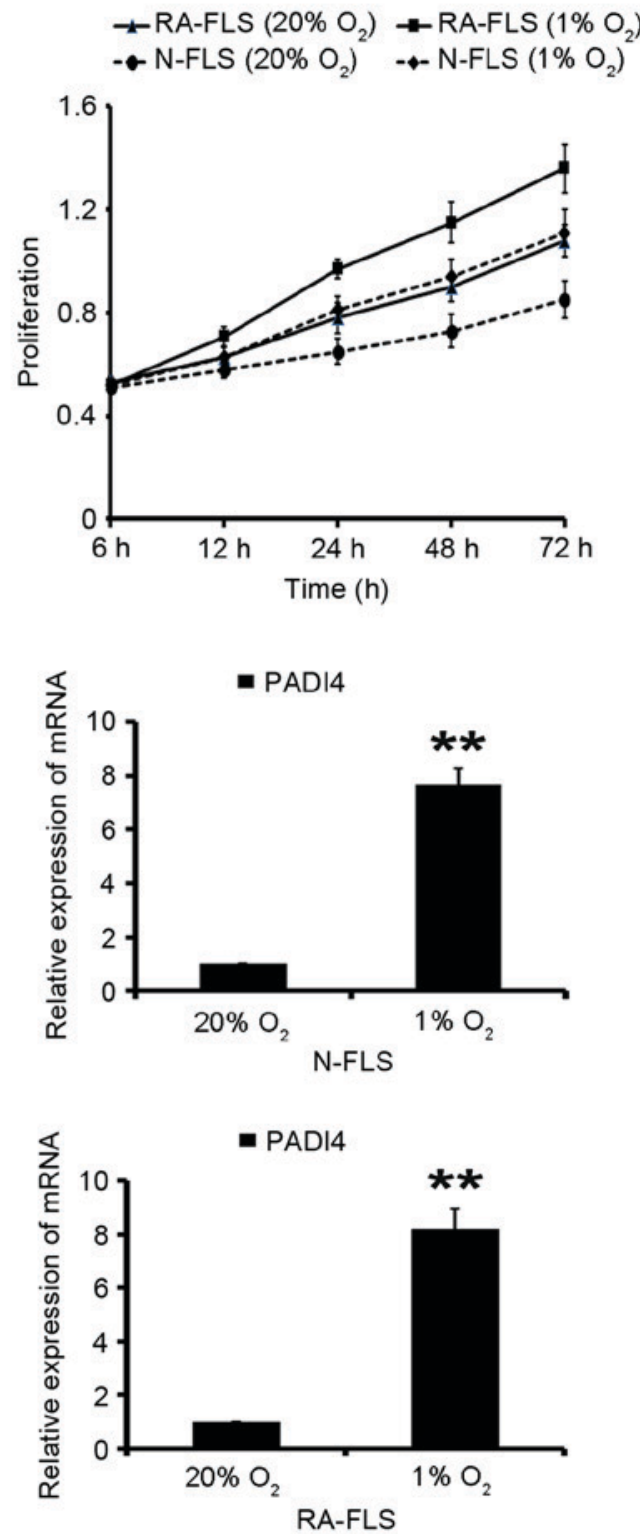

$\mathrm{E}$

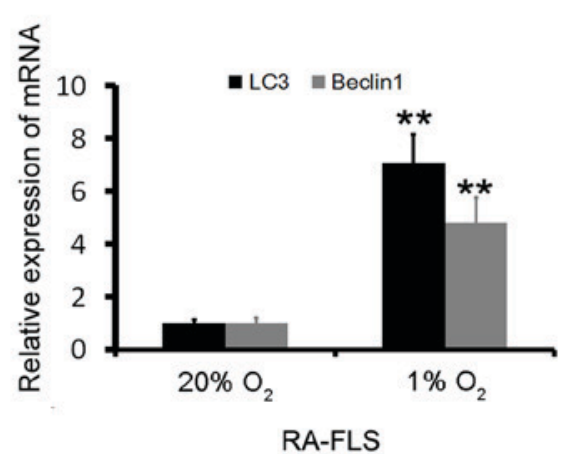

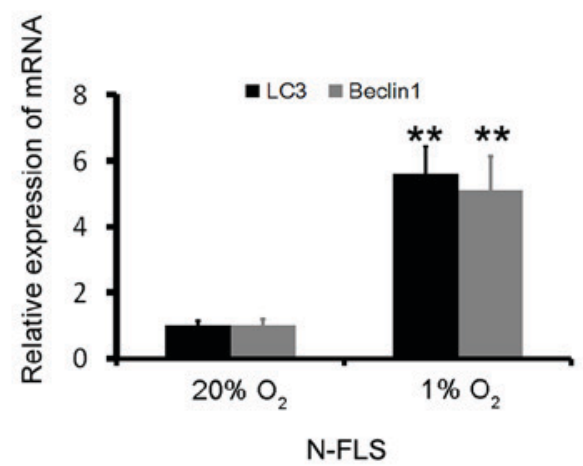

Figure 3. PADI4 promotes the proliferation of RA-FLS through hypoxia. (A) RA-FLS and N-FLS were incubated under normoxia $\left(20 \% \mathrm{O}_{2}\right)$ or hypoxia $\left(1 \% \mathrm{O}_{2}\right)$ for 5 days. (B) Cell viability under normoxia and hypoxia was measured using an MTT assay. Data are presented as mean \pm standard deviation from three separate experiments. (C) PADI4 expression was determined by immunofluorescence and qPCR in N-FLS under normoxia and hypoxia for 3 days. (D) PADI4 expression was determined by immunofluorescence and qPCR in RA-FLS under normoxia and hypoxia for 3 days. (E) LC3 and Beclin1 expression were determined by qPCR in RA-FLS under normoxia and hypoxia for 3 days. N-FLS were obtained from control rats. Data represent three independent experiments with presented as mean \pm standard deviation. ${ }^{* *} \mathrm{P}<0.001$. FLS, fibroblast-like synoviocytes; PADI4, peptidyl arginine deiminase type IV; qPCR, quantitative polymerase chain reaction; RA, rheumatoid arthritis.

induction of autophagy was identified by two well-established measurements of autophagy; the enhancement of Beclin1, a component of the class III phosphatidylinositol 3-kinase complex essential for autophagosome formation, and LC3 (16) 
A
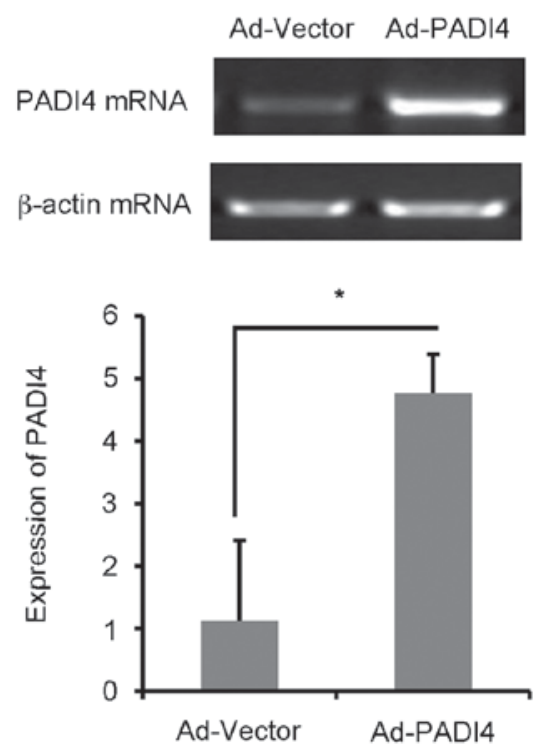

B
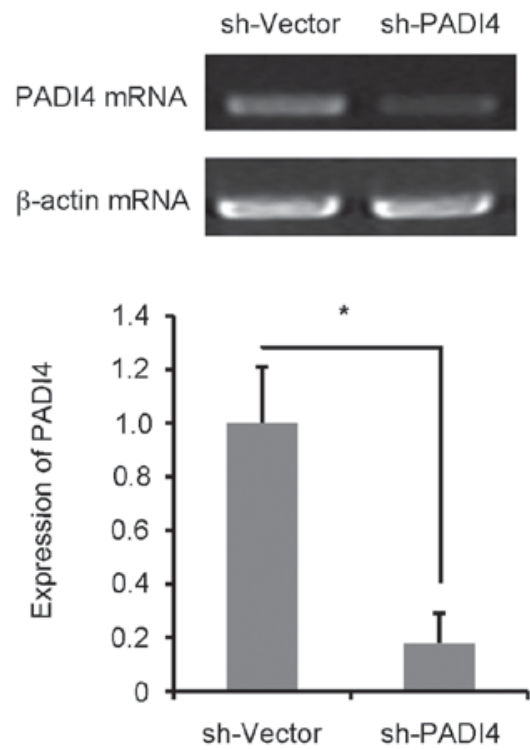

Figure 4. PADI4 expression in FLS. Post-transfection of (A) N-FLS with Ad-PADI4 and (B) RA-FLS with sh-PADI4, the mRNA expression levels of PADI4 were measured by reverse transcription-quantitative polymerase chain reaction. Data represent three independent experiments with presented as mean \pm standard deviation. "P<0.01. Ad, adenovirus; FLS, fibroblast-like synoviocytes; PADI4, peptidyl arginine deiminase type IV; RA, rheumatoid arthritis; sh, short hairpin RNA.

as presented in Fig. 3E. In order to investigate the effects of PADI4 on hypoxia-induced autophagy in FLS, N-FLS transfected with Ad-PADI4 and RA-FLS transfected with PADI4 shRNA were cultured under hypoxia for 5 days.

FLS with Ad-PADI4 or sh-PADI4 transfection were examined by RT-qPCR (Fig. 4A and B). Ad-PADI4 transfection significantly increased the mRNA expression levels of PADI4 in N-FLS and sh-PADI4 transfection significantly decreased the mRNA expression levels of PADI4 in RA-FLS. FLS with GFP-LC3 plasmid transfection were examined under hypoxia using a fluorescent microscope (Fig. 5A). RA-FLS with PADI4 shRNA exhibited a diffuse expression pattern of GFP-LC3, whereas N-FLS with Ad-PADI4 revealed a punctate pattern of GFP-LC3, indicating the formation of autophagosomes. Furthermore, a large number of N-FLS transfected with Ad-PADI4 exhibited increased LC3 and Beclin1 expression, compared with RA-FLS transfected with PADI4 shRNA (Fig. 5B-E). These data suggested that ectopic expression of PADI4, induced by transfection of N-FLS with PADI4 expression plasmid, may be involved in hypoxia-induced autophagy. Consistent with these results, PADI4 inactivation partially inhibited hypoxia-induced autophagy.

PADI4 inactivation induces RA-FLS apoptosis. The results of the present study indicated that FLS proliferation is increased under hypoxia. Subsequently, the study aimed to determine the effects of PADI4 on FLS under hypoxia. After being cultured under hypoxia for 3 days N-FLS transfected with Ad-PADI4 exhibited significant proliferation compared with N-FLS transfected with Ad-vector; whereas the growth of RA-FLS with PADI4 shRNA was suppressed compared with RA-FLS transfected with sh-vector (Fig. 6A). Apoptosis of FLS was analyzed by flow cytometry. The results demonstrated that apoptosis was enhanced in RA-FLS transfected with PADI4 shRNA, thus suggesting that PADI4 is an important factor in the inhibition of RA-FLS apoptotic death (Fig. 6B). These data suggested that PADI4 inactivation may be involved in hypoxia-induced autophagy, and may induce RA-FLS apoptosis.

\section{Discussion}

RA is a chronic and complex autoimmune disease with a complex etiology, which leads to generalized bone loss, cartilage erosion and increased fracture risk. Although the causes of RA remain unclear, it has been reported to be attributed to genetics, autoimmunity and lifestyle-associated factors (17). RA-FLS serve a crucial role in producing cytokines that are responsible for inflammation, and proteases that may result in cartilage destruction. RA-FLS are associated with the initiation and perpetuation of RA, in which impaired apoptosis of RA-FLS is pivotal; however, the molecular mechanism underlying reduced apoptosis remains to be elucidated (10).

The PADI4 gene, which encodes the PADI4 enzyme, has an important role in protein citrullination, which is a key event underlying the pathogenesis of RA. PADI4 is involved in gene regulation through the citrullination of histones, which contributes to the generation of ACPA specific substrates, and is itself a target of autoantibodies in RA (2). PADI4 polymorphisms represent a significant risk factor for RA not only in Asian populations, but also in populations of European descent $(18,19)$. Furthermore, increased transcription of PADI4 has been detected in the synovial membrane of RA (20). Our previous results indicated that citrullinated vimentin significantly increased the expression of PADI4 in cultured RA-FLS (21). The present study demonstrated that PADI4 protein expression was elevated in human RA-FLS. Subsequently, the study aimed to determine whether PADI4 could be associated with the impaired apoptosis of RA-FLS. 
A

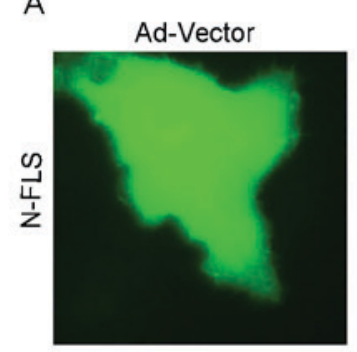

sh-vector
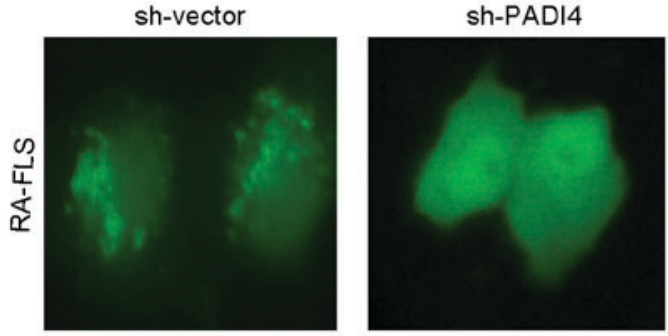

B
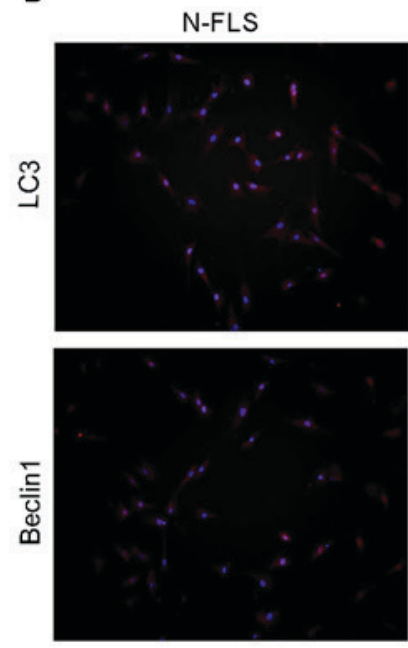

D
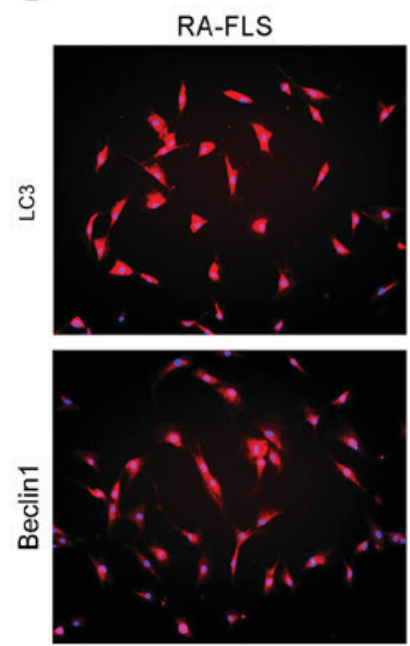

Ad-PADI4

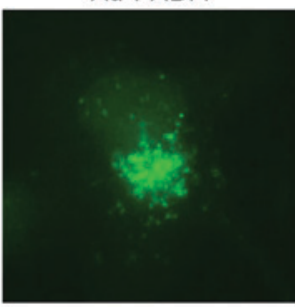

sh-PADI4

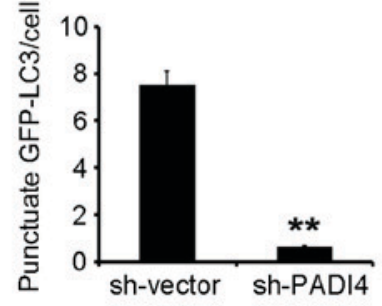

N-FLS+Ad-Vector
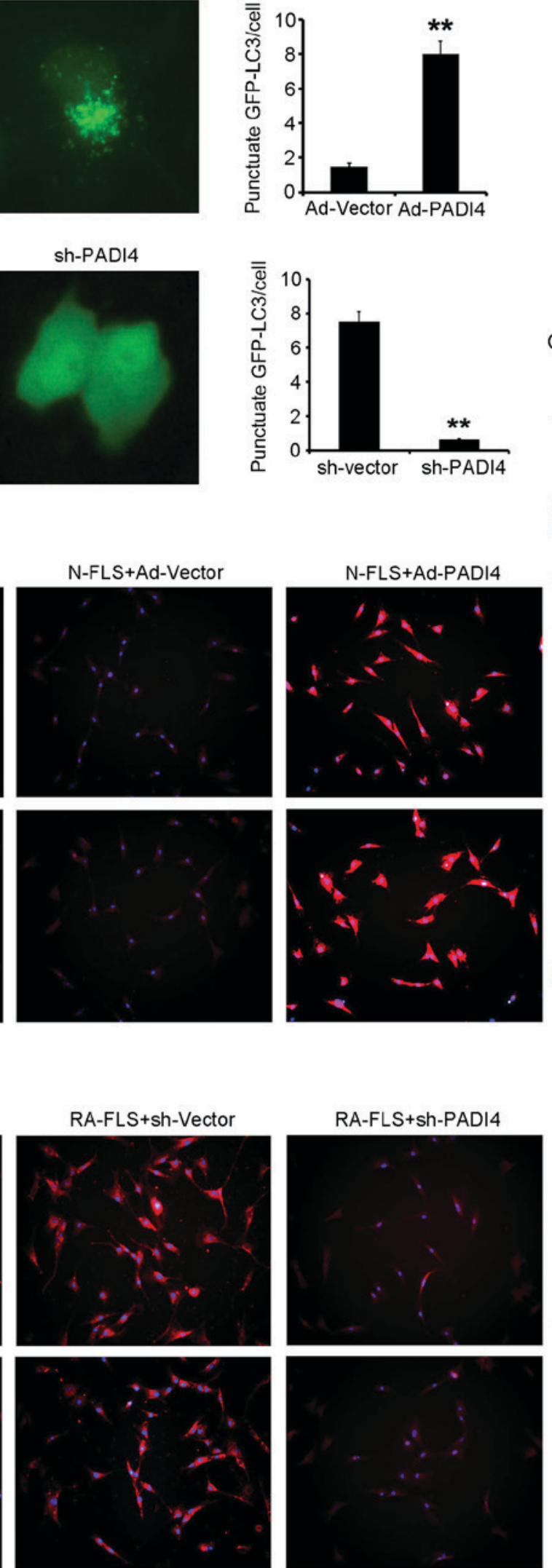

C
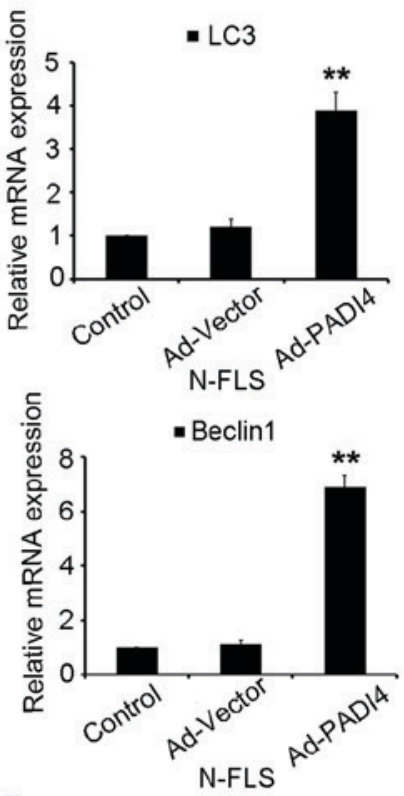

E

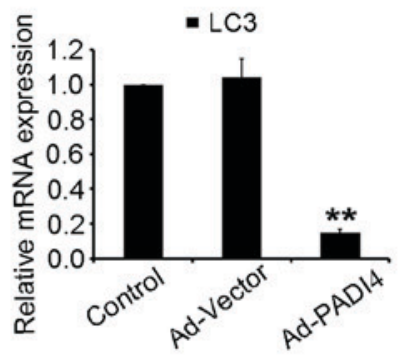

RA-FLS

- Beclin1

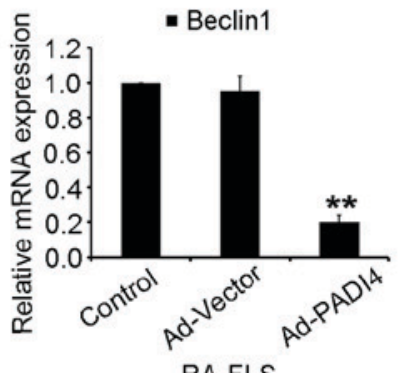

Figure 5. Inactivation of PADI4 partially inhibits hypoxia-induced autophagy. (A) Representative images (magnification, x1,000) of GFP-LC3 expression patterns in FLS transfected with Ad-PADI4 orsh-PADI4 under hypoxia $\left(1 \% \mathrm{O}_{2}\right)$ for 3 days. FLS transfected with GFP-LC3 plasmid were detected. Expression of LC3 and Beclin1 detected using (B) immunofluorescence (magnification, x400) and (C) qPCR in N-FLS transfected with Ad-PADI4 under hypoxia for 3 days. Expression of LC3 and Beclin1 detected by (D) immunofluorescence (magnification, $\mathrm{x} 400$ ) and (E) qPCR in RA-FLS transfected with sh-PADI4 under hypoxia for 3 days. Data represent three independent experiments with presented as mean \pm standard deviation. ${ }^{* *} \mathrm{P}<0.001$. Ad, adenovirus; FLS, fibroblast-like synoviocytes; GFP, green fluorescent protein; LC3, microtubule-associated light chain 3; PADI4, peptidyl arginine deiminase type IV; RA, rheumatoid arthritis; sh, short hairpin RNA. 
A
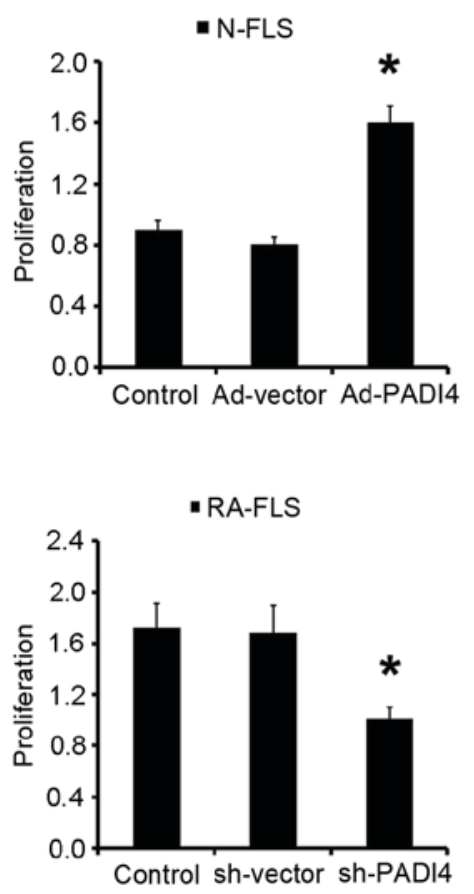

B
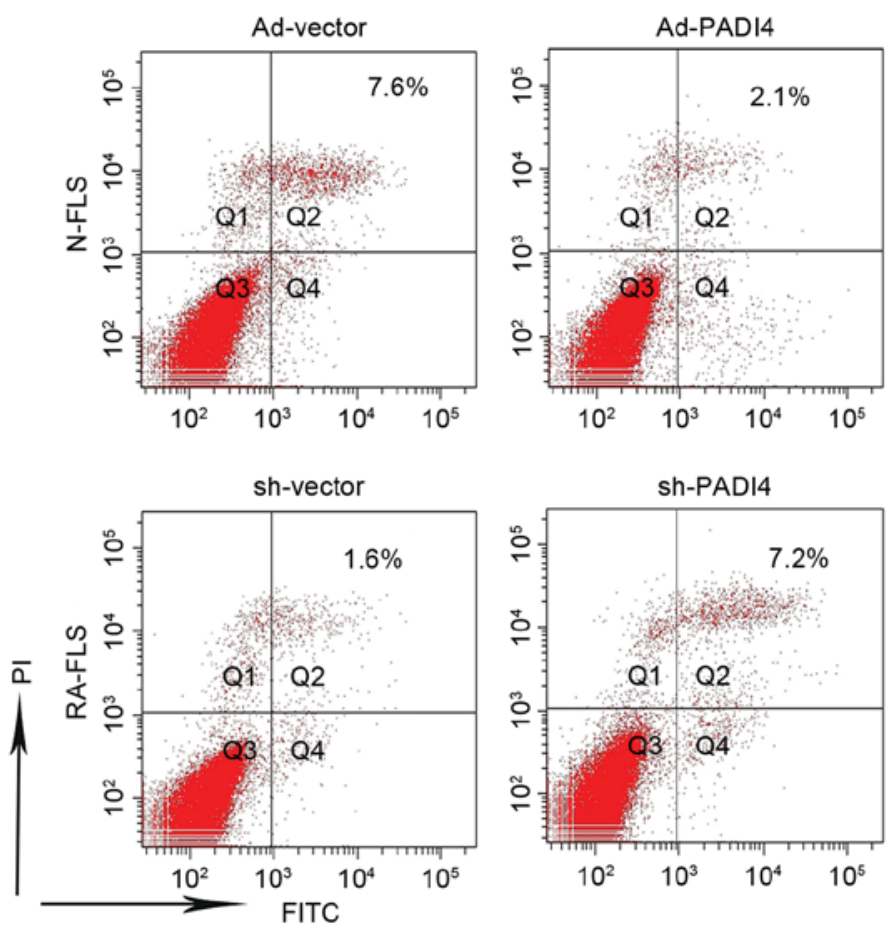

Figure 6. PADI4 inactivation induces FLS apoptosis. (A) RA-FLS transfected with sh-PADI4 and N-FLS transfected with Ad-PADI4 were cultured under hypoxia $\left(1 \% \mathrm{O}_{2}\right)$ for 3 days. Cell viability was measured by MTT. (B) FLS were stained with Annexin V-FITC (20 mg/ml) and PI (20 mg/l) under hypoxia for 5 days. Apoptosis was analyzed using flow cytometry. Data represent 3 independent experiments with presented as mean \pm standard deviation. ${ }^{*} \mathrm{P}<0.01$. Ad, adenovirus; FITC, fluorescein isothiocyanate; FLS, fibroblast-like synoviocytes; PADI4, peptidyl arginine deiminase type IV; PI, propidium iodide; RA, rheumatoid arthritis; sh, short hairpin RNA.

Hypoxia is a key regulator of angiogenesis and inflammation in RA (22). HIF has been reported to induce adaptive responses to hypoxic stress by activating a large number of genes that are responsible for oxygen delivery, angiogenesis, cell proliferation, cell differentiation and metabolism (23). The present study revealed that HIF- $1 \alpha$ protein expression was elevated in human RA-FLS, thus indicating that hypoxia may be a factor promoting the proliferation of RA-FLS. In addition, it has been reported that synovial tissue was hypoxic in patients with RA compared with in noninflamed synovium from patients without RA (24). Autophagy has been reported to be induced by hypoxia (25). Hypoxic stress, which is mediated through HIF-1 $\alpha$, is a strong signal that initiates the autophagic process (26).

HIF-1 $\alpha$ has been reported to serve a critical role in the regulation of hypoxia-induced angiogenesis (27). In addition, autophagy modulates apoptosis; it has previously been demonstrated that autophagy inhibits apoptosis through the degradation of proapoptotic proteins, including caspases (28). In the present study, PADI4 overexpression in RA rat model may have promoted the proliferation of RA-FLS.

It has been hypothesized that inhibition of autophagy promotes apoptosis in cancer cells with intact apoptotic signaling pathways (29). Furthermore, enhanced autophagy was associated with elevated levels of HIF-1 $\alpha$. In the present study, IHC revealed that LC3 and Atg5 were overexpressed in resected synovial specimens obtained from patients with RA compared with in tissues obtained from patients with OA. These findings indicated that hypoxia-induced autophagy may serve an important role in synovial tissues.
The number of RA-FLS that originated from the RA rat model markedly increased in the presence of $1 \% \mathrm{O}_{2}$, but not in normal oxygen conditions. In addition, numerous stressors, including severe hypoxia or oxidative stress could induce activation of autophagy in synovial tissues obtained from patients with RA (30). In the present study, after being cultured under hypoxic conditions, a marked increase in PADI4 expression was observed in cultured RA-FLS. Furthermore, a significant increase in Beclin1 and LC3 expression was detected in cells with ectopic overexpression of PADI4. Conversely, decreased Beclin1 and LC3 expression was detected in cells transfected with PADI4 shRNA. Increased apoptosis of RA-FLS was also observed in response tosh-PADI4 transfection, thus indicating that suppression of RA-FLS may be associated with PADI4 inhibition. The in vivo and in vitro results of the present study suggested that PADI4 may be closely associated with hypoxia-induced autophagy.

In conclusion, these results revealed that inactivation of hypoxia-induced autophagy via the knockdown of PADI4 may contribute to increased apoptosis of RA-FLS. Therefore, further studies that investigate the role of PADI4 in hypoxia-induced autophagy may provide novel information regarding the mechanism underlying the impaired apoptosis of RA-FLS. The present study also proposed a novel mechanism underlying the close association between hypoxia, autophagy and inflammation in RA, and supported the concept that autophagic inhibition may be of therapeutic benefit in RA; however, the present study had some shortcomings. The number of patients enrolled was insufficient and the effects of PADI4 on the growth of RA-FLS were determined using 
immunostaining only. Therefore, the findings of the present study should be further investigated in future studies.

\section{Acknowledgements}

The present study was supported by the Natural Science Foundation of China (grant nos. 81373203 and 81372212), the Shanghai Municipal Science and Technology Commission (grant no. 12XD1404300) and the Natural Science Foundation of Jiangsu (grant nos. BK2011251 and BL2013012).

\section{Competing interests}

The authors declare that they have no competing interests.

\section{References}

1. Ikari K, Yano K, Yoshida S, Taniguchi A, Yamanaka H and Momohara S: Response to 'Peptidyl arginine deiminase type IV (PADI4) haplotypes interact with shared epitope regardless of anti-cyclic citrullinated peptide antibody or erosive joint status in rheumatoid arthritis: A case control study'. Arthritis Res Ther 16: 422, 2014.

2. Dong S, Zhang Z and Takahara H: Estrogen-enhanced peptidylarginine deiminase type IV gene (PADI4) expression in MCF-7 cells is mediated by estrogen receptor-alpha-promoted transfactors activator protein-1, nuclear factor-Y, and Sp1. Mol Endocrinol 21: 1617-1629, 2007.

3. Yamada R, Suzuki A, Chang X and Yamamoto K: Citrullinated proteins in rheumatoid arthritis. Front Biosci 10: 54-64, 2005.

4. Suzuki A, Yamada R, Chang X, Tokuhiro S, Sawada T, Suzuki M, Nagasaki M, Nakayama-Hamada M, Kawaida R, Ono M, et al: Functional haplotypes of PADI4, encoding citrullinating enzyme peptidylarginine deiminase 4 , are associated with rheumatoid arthritis. Nat Genet 34: 395-402, 2003.

5. Zavala-Cerna MG, Gonzalez-Montoya NG, Nava A, Gamez-Nava JI, Moran-Moguel MC, Rosales-Gomez RC, Gutierrez-Rubio SA Sanchez-Corona J, Gonzalez-Lopez L, Davalos-Rodriguez IP and Salazar-Paramo M: PADI4 haplotypes in association with RA Mexican patients, a new prospect for antigen modulation. Clin Dev Immunol 2013: 383681, 2013.

6. Bang SY, Han TU, Choi CB, Sung YK, Bae SC and Kang C: Peptidyl arginine deiminase type IV (PADI4) haplotypes interact with shared epitope regardless of anti-cyclic citrullinated peptide antibody or erosive joint status in rheumatoid arthritis: A case control study. Arthritis Res Ther 12: R115, 2010.

7. Suzuki T, Ikari K, Yano K, Inoue E, Toyama Y, Taniguchi A, Yamanaka $\mathrm{H}$ and Momohara S: PADI4 and HLA-DRB1 are genetic risks for radiographic progression in RA patients, independent of ACPA status: Results from the IORRA cohort study. PLoS One 8: e61045, 2013.

8. Bartok B and Firestein GS: Fibroblast-like synoviocytes: Key effector cells in rheumatoid arthritis. Immunol Rev 233: 233-255, 2010.

9. Bottini N and Firestein GS: Duality of fibroblast-like synoviocytes in RA: Passive responders and imprinted aggressors. Nat Rev Rheumatol 9: 24-33, 2013.

10. Li H and Wan A: Apoptosis of rheumatoid arthritis fibroblast-like synoviocytes: Possible roles of nitric oxide and the thioredoxin 1. Mediators Inflamm 2013: 953462, 2013.

11. Gu X, Gu B, Lv X, Yu Z, Wang R, Zhou X, Qiao W, Mao Z, Zuo G, Li Q, et al: 1, 25-dihydroxy-vitamin D3 with tumor necrosis factor-alpha protects against rheumatoid arthritis by promoting p53 acetylation-mediated apoptosis via Sirt1 in synoviocytes. Cell Death Dis 7: e2423, 2016.

12. Wang L, Zhang HY, Gao B, Shi J, Huang Q, Han YH, Hu YQ, Lu WG, Zhao ZJ, Liu BH, et al: Tetramethylpyrazine protects against glucocorticoid-induced apoptosis by promoting autophagy in mesenchymal stem cells and improves bone mass in glucocorticoid-induced osteoporosis rats. Stem Cells Dev 26: 419-430, 2017.
13. Fan L, Wang Q, Liu R, Zong M, He D, Zhang H, Ding Y and Ma J: Citrullinated fibronectin inhibits apoptosis and promotes the secretion of pro-inflammatory cytokines in fibroblast-like synoviocytes in rheumatoid arthritis. Arthritis Res Ther 14: R266, 2012.

14. Lin TN, He YY, Wu G, Khan M and Hsu CY: Effect of brain edema on infarct volume in a focal cerebral ischemia model in rats. Stroke 24: 117-121, 1993.

15. Livak KJ and Schmittgen TD: Analysis of relative gene expression data using real-time quantitative PCR and the 2(-Delta Delta C(T)) method. Methods 25: 402-408, 2001.

16. Matsunaga K, Saitoh T, Tabata K, Omori H, Satoh T, Kurotori N, Maejima I, Shirahama-Noda K, Ichimura T, Isobe T, et al: Two Beclin 1-binding proteins, Atg14L and Rubicon, reciprocally regulate autophagy at different stages. Nat Cell Biol 11: 385-396, 2009.

17. Kremer JM: Rheumatoid arthritis: New EULAR guidelines for RA: A job well done. Nat Rev Rheumatol 10: 6-8, 2014.

18. Hou S, Gao GP, Zhang XJ, Sun L, Peng WJ, Wang HF, Ge XJ, Huang W and Sun YH: Erratum to: PADI4 polymorphisms and susceptibility to rheumatoid arthritis: A meta-analysis. Mod Rheumatol 23: 61, 2013.

19. Iwamoto T, Ikari K, Nakamura T, Kuwahara M, Toyama Y, Tomatsu T, Momohara S and Kamatani N: Association between PADI4 and rheumatoid arthritis: A meta-analysis. Rheumatology (Oxford) 45: 804-807, 2006.

20. Chang X, Zhao Y, Sun S, Zhang Y and Zhu Y: The expression of PADI4 in synovium of rheumatoid arthritis. Rheumatol Int 29: 1411-1416, 2009.

21. Reyes-Castillo Z, Palafox-Sánchez CA, Parra-Rojas I, Martínez-Bonilla GE, del Toro-Arreola S, Ramírez-Dueñas MG, Ocampo-Bermudes G and Muñoz-Valle JF: Comparative analysis of autoantibodies targeting peptidylarginine deiminase type 4 , mutated citrullinated vimentin and cyclic citrullinated peptides in rheumatoid arthritis: Associations with cytokine profiles, clinical and genetic features. Clin Exp Immunol 182: 119-131, 2015.

22. Konisti S, Kiriakidis S and Paleolog EM: Hypoxia-a key regulator of angiogenesis and inflammation in rheumatoid arthritis. Nat Rev Rheumatol 8: 153-162, 2012.

23. Brouwer E, Gouw AS, Posthumus MD, van Leeuwen MA, Boerboom AL, Bijzet J, Bos R, Limburg PC, Kallenberg CG and Westra J: Hypoxia inducible factor-1-alpha (HIF-1alpha) is related to both angiogenesis and inflammation in rheumatoid arthritis. Clin Exp Rheumatol 27: 945-951, 2009.

24. Muz B, Khan MN, Kiriakidis S and Paleolog EM: Hypoxia. The role of hypoxia and HIF-dependent signalling events in rheumatoid arthritis. Arthritis Res Ther 11: 201, 2009.

25. Marin JJG, Lozano E and Perez MJ: Lack of mitochondrial DNA impairs chemical hypoxia-induced autophagy in liver tumor cells through ROS-AMPK-ULK1 signaling dysregulation independently of HIF-1 $\alpha$. Free Radic Biol Med 101: 71-84, 2016.

26. Zhang Z, Yang M, Wang Y, Wang L, Jin Z, Ding L, Zhang L, Zhang L, Jiang W, Gao G, et al: Autophagy regulates the apoptosis of bone marrow-derived mesenchymal stem cells under hypoxic condition via AMP-activated protein kinase/mammalian target of rapamycin pathway. Cell Biol Int 40: 671-685, 2016.

27. Park SY, Jang WJ, Yi EY, Jang JY, Jung Y, Jeong JW and Kim YJ: Melatonin suppresses tumor angiogenesis by inhibiting HIF-1alpha stabilization under hypoxia. J Pineal Res 48: 178-184, 2010.

28. Feng LX, Sun P, Mi T, Liu M, Liu W, Yao S, Cao YM, Yu XL, Wu WY, Jiang BH, et al: Agglutinin isolated from Arisema heterophyllum Blume induces apoptosis and autophagy in A549 cells through inhibiting PI3K/Akt pathway and inducing ER stress. Chin J Nat Med 14: 856-864, 2016.

29. Ouyang L, Shi Z, Zhao S, Wang FT, Zhou TT, Liu B and Bao JK: Programmed cell death pathways in cancer: A review of apoptosis, autophagy and programmed necrosis. Cell Prolif 45: 487-498, 2012.

30. Kato M, Ospelt C, Gay RE, Gay S and Klein K: Dual role of autophagy in stress-induced cell death in rheumatoid arthritis synovial fibroblasts. Arthritis Rheumatol 66: 40-48, 2014.

This work is licensed under a Creative Commons Attribution-NonCommercial-NoDerivatives 4.0 International (CC BY-NC-ND 4.0) License. 